\title{
Indications requiring preoperative magnetic resonance imaging before knee arthroscopy
}

\author{
Björn Peter Roßbach ${ }^{1}$, Matthias Frank Pietschmann ${ }^{1}$, Mehmet Fatih Gülecyüz ${ }^{1}$, \\ Thomas Richard Niethammer ${ }^{1}$, Andreas Ficklscherer ${ }^{1}$, Stefan Wild ${ }^{2}$, Volkmar Jansson ${ }^{1}$, \\ Peter Ernst Müller ${ }^{1}$
}

\author{
${ }^{1}$ Department of Orthopaedic Surgery, University Hospital of Munich (LMU), Munich, \\ Germany \\ ${ }^{2}$ Department of Trauma, Hand and Reconstructive Surgery, Academic Hospital \\ of Munich, Klinikum Augsburg, Augsburg, Germany
}

Submitted: 29 April 2013

Accepted: 15 June 2013

Arch Med Sci 2014; 10, 6: 1147-1152

DOI: $10.5114 /$ aoms.2014.47825

Copyright $\odot 2014$ Termedia \& Banach

\section{Abstract}

Introduction: Knee arthroscopy knee is gold standard in diagnosis and simultaneous treatment of knee disorders. But most patients undergo magnetic resonance imaging (MRI) before arthroscopy, although MRI results are not always consistent with arthroscopic findings. This raises the question in which suspected diagnoses MRI really has influence on diagnosis and consecutive surgical therapy.

Material and methods: Preoperative MRI of 330 patients with knee disorders were compared with arthroscopic findings. The MRI were performed by 23 radiologists without specialization in musculoskeletal diagnostics. Specificity, sensitivity, negative/positive predictive value and accuracy of MRI were calculated in comparison to arthroscopic findings.

Results: We found sensitivity/specificity of $58 \% / 93 \%$ for anterior horn, $94 \% / 46 \%$ for posterior horn of medial meniscus and $71 \% / 81 \%$ for anterior and $62 \% / 82 \%$ for posterior horn of lateral meniscus. Related to anterior cruciate ligament injuries we showed sensitivity/specificity of $82 \% / 91 \%$ for grade $0+\mathrm{I}$ and $72 \% / 96 \%$ for grade II + III. For Cartilage damage sensitivity/specificity of $98 \% / 7 \%$ for grade I-, $89 \% / 29 \%$ for grade $11-, 96 \% / 38 \%$ for grade III- and $96 \% / 69 \%$ for grade IV-lesions were revealed.

Conclusions: The MRI should not be used as routine diagnostic tool for knee pain. No relevant information for meniscal lesions and anterior cruciate ligament ruptures has been gained with MRI from non-specialized outside imaging centres.

Key words: magnetic resonance imaging, arthroscopy, knee, specificity, sensitivity, diagnostic tool.

\section{Introduction}

With worldwide approximately 3.5 million procedures per year, arthroscopy of the knee is the most common orthopaedic surgery procedure [1]. Although knee arthroscopy is now considered the gold standard in diagnosis and simultaneous treatment of knee disorders, most patients undergo magnetic resonance imaging (MRI) before arthroscopic treatment [2, 3]. Neither arthroscopy nor MRI guarantees 100 percent detection of knee pathologies, but both procedures can distinguish pathological
Corresponding author: Prof. Dr. med. Peter E. Müller Department of Orthopaedic Surgery University Hospital of Munich (LMU) Campus Grosshadern Marchioninistr. 15 81377 Munich, Germany Phone: +49 89440073781 Fax: +49 89440076780 E-mail: Peter.Mueller@ med.uni-muenchen.de 
changes with a respectable value. There are numerous studies with excellent results regarding sensitivity and specificity of MRI diagnosis of meniscal tears, ligament injuries and cartilage damage [4-10]. Magnetic resonance imaging is an advantage in diagnosis of meniscus lesions, particularly in the early detection of grade I and grade Il lesions, definition of a surgical intervention and postoperative follow-up [11]. Furthermore, MRI is seen as an important tool especially in diagnosis and grading of cartilage injuries [12]. In addition to that, knee MRI is useful not only in chronic clinical presentations, but also in acute traumatic extension deficits in the acute or subacute phase in patients having a mechanical reason for a "locked knee" and would benefit from arthroscopic treatment [13].

However, these data were always collected in specialized units for musculoskeletal radiology, but we consider that they are not comparable with data from MRI scans of non-specialized units. Most surgeons indeed receive many different MRI data from outpatient radiology units without specialization in musculoskeletal diagnostics and not from a single specialized unit with a standardized high-resolution MR scanner.

In the literature, many studies state that preoperative MRI should be performed on principle to avoid surgical risks of arthroscopy and to reduce the rate of unnecessary diagnostic arthroscopies $[2,6,7,9,14]$. On the other hand, there are some studies pointing out that MRI is not routinely necessary for the indication of knee arthroscopy and that it should only be used as a diagnostic tool to rule out injuries $[4,15]$. Additionally, MRI results are not always consistent with arthroscopic findings [16]. With the current widespread use of preoperative $\mathrm{MRI}$, some pathologies seen on MRI have proven to represent true pathology, while others have been shown to be normal variants or artefacts in arthroscopy [17-20].

This raises the question with which suspected diagnoses it makes sense, and with which sus-

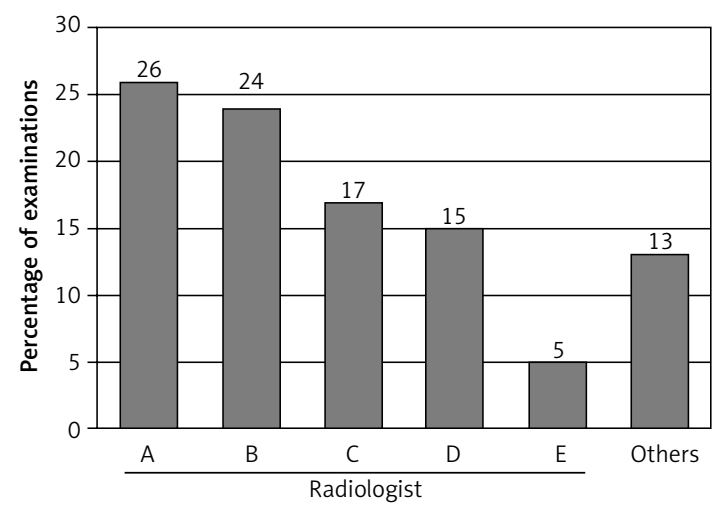

Figure 1. Number of radiologists without musculoskeletal specialization generating the MRI reports pected diagnoses it makes no sense, to perform preoperative MRI, and also with which suspected diagnoses MRI really has an influence on diagnosis und consecutive surgical therapy raised by clinical examination.

We therefore hypothesized that knee MRI in a radiologic unit without musculoskeletal specialization provides no relevant information gain regarding certain questions.

\section{Material and methods}

Over a time period of 31 months, 330 patients with a sex ratio of 169 males to 161 females and 181 right to 149 left knee joints were examined in one clinic and underwent knee arthroscopy by an experienced senior surgeon after MRI scan. Chronic knee disorders were present in 227 patients and 103 patients presented an acute trauma as a knee distortion or contusion. Patients' average age was 52.1 years (range 14 to 82 years). Patients with prior surgery were excluded from this study.

Preoperative MRI scans of all patients were performed and evaluated by a total of 23 radiologists in outpatient radiology units without specialization in musculoskeletal diagnostics (Figure 1). Radiologists did not assess the MRI findings of their colleagues again. Closed MR scanners with a magnetic flux density of 1.5 Tesla were used in all cases. Standard imaging sequences at outside imaging centres included coronal T1-weighted sequence and proton-density turbo spin-echo fat-suppressed sequences for sagittal, axial and coronal planes.

The MRI report was known to the surgeon before arthroscopy. After the surgical procedure, pathological findings were compared with the preoperative MRI report. The study was approved by the ethics committee of the university.

\section{Assessment of pathological findings in MRI scans and arthroscopy}

\section{Meniscus}

Table I shows the grading of meniscal lesions in direct comparison between MRI and arthroscopy [21].

\section{Anterior cruciate ligament}

The grading for disorders of the anterior cruciate ligament in direct comparison between MRI and arthroscopy is shown in Table II.

\section{Cartilage}

To evaluate arthroscopic cartilage damage, the classification of the International Cartilage Repair Society (ICRS) in accordance with the Outerbridge classification was used [22]. For classification of cartilage lesions on MRI a modified Outerbridge 
Table I. Modified grading of meniscal lesions [21]

\begin{tabular}{|lcc|}
\hline Grade & MRI & Arthroscopy \\
\hline 0 & Intact & Intact \\
\hline I & Degeneration & Degeneration \\
\hline II & Rupture & Rupture \\
\hline III & Status post partial resection & Status post partial resection \\
\hline IV & Status post partial resection with re-rupture & Status post partial resection with re-rupture \\
\hline V & Tear-off of intermediate meniscal part & Tear-off of intermediate meniscal part \\
\hline
\end{tabular}

classification was applied [23]. This allowed a direct comparison between $\mathrm{MRI}$ reports and arthroscopic findings (Table III).

Five joint surfaces of every patient's knee were evaluated. In detail this was the femoral and the tibial joint surface of the lateral and medial compartment. The patellar joint surface of the femur and back side of the patella were combined as the retropatellar surface. This disposition was provided by the radiologists, who did not make any distinction between the articular surface of the femur and back side of the patella. A total of 1650 joint surfaces were evaluated in 330 patients.

\section{Statistical analysis}

To allow comparison with other studies, specificity, sensitivity, negative/positive predictive value and accuracy of MRI in comparison with arthroscopic findings were calculated. Data were processed using the SPSS software (IBM SPSS Statistics 19.0) for Microsoft Windows.

Sensitivity of a medical test is defined as the proportion of people who have a disease and are also tested positive for it. Specificity is defined as the proportion of patients who do not have the disease and who are tested negative for it. Negative/positive predictive value is the proportion of subjects with a negative/positive test result, who are correctly diagnosed. Accuracy is the degree of closeness of measurements of a quantity to that quantity's actual value.
Table II. Modified grading for disorders of the anterior cruciate ligament [21]

\begin{tabular}{|lcc|}
\hline Grade & MRI & Arthroscopy \\
\hline 0 & Intact & Intact \\
\hline I & $\begin{array}{c}\text { Signs of } \\
\text { degeneration }\end{array}$ & $\begin{array}{c}\text { Signs of } \\
\text { degeneration }\end{array}$ \\
\hline II & Partial rupture & Partial rupture \\
\hline III & Complete rupture & Complete rupture \\
\hline
\end{tabular}

\section{Results}

\section{Meniscus}

With regards to localization, the highest specificity was seen for the anterior horn of the medial meniscus and the lowest specificity for the posterior horn of the medical meniscus (Table IV). Approximately the same specificity was seen for the anterior and posterior horn of the lateral meniscus.

The highest sensitivity was observed for the posterior horn of the medial meniscus and the lowest for the anterior horn of the medial meniscus. The anterior and posterior horn of the lateral meniscus showed similar values regarding sensitivity. High specificity and low sensitivity for the anterior horn of the medial meniscus may be related to the poor visibility of the anterior horn by the conventional approach.

Table III. Modified grading for cartilage lesions in MRI and arthroscopy

\begin{tabular}{|c|c|c|}
\hline Grade & MRI (Uhl et al.) [23] & Arthroscopy (ICRS) [22] \\
\hline 0 & Normal & Normal \\
\hline I & $\begin{array}{c}\text { Hypo- or hyper-signal, surface roughness, } \\
\text { chondromalacia }\end{array}$ & $\begin{array}{c}\text { Loss of elasticity, surface roughness, } \\
\text { chondromalacia }\end{array}$ \\
\hline II & $\begin{array}{l}\text { Small surface irregularities, } \\
\text { focal depth reduction }<50 \%\end{array}$ & $\begin{array}{l}\text { Damage of cartilage surface, } \\
\text { lesion }<50 \% \text { of depth }\end{array}$ \\
\hline III & $\begin{array}{l}\text { Significant surface irregularities, } \\
\text { focal depth reduction }>50 \%\end{array}$ & $\begin{array}{l}\text { Damage of cartilage surface, } \\
\text { lesion }>50 \% \text { of depth, bone is not exposed }\end{array}$ \\
\hline IV & $\begin{array}{l}\text { Focal } 100 \% \text { depth reduction, } \\
\text { subchondral bone is exposed }\end{array}$ & Bone is exposed \\
\hline
\end{tabular}


Table IV. Statistical data for meniscal lesions related to localization (grade I-IV; in \%)

\begin{tabular}{|lcccc|}
\hline Parameter & $\begin{array}{c}\text { Anterior horn } \\
\text { of medial meniscus }\end{array}$ & $\begin{array}{c}\text { Posterior horn } \\
\text { of medial meniscus }\end{array}$ & $\begin{array}{c}\text { Anterior horn } \\
\text { of lateral meniscus }\end{array}$ & $\begin{array}{c}\text { Posterior horn } \\
\text { of lateral meniscus }\end{array}$ \\
\hline Specificity & 93.1 & 46.2 & 80.8 & 82.3 \\
\hline Sensitivity & 58.3 & 94.4 & 70.5 & 62.2 \\
\hline Negative predictive value & 98.3 & 81.8 & 94.7 & 56.8 \\
\hline Positive predictive value & 24.1 & 76.1 & 36.0 & 79.4 \\
\hline Accuracy & 91.8 & 77.3 & 77.3 \\
\hline
\end{tabular}

\section{Anterior cruciate ligament}

Grade $0+$ I as well as grade II + III lesions of the anterior cruciate ligament showed higher specificity than sensitivity. The high negative predictive value for all four damage levels is striking (Table V).

\section{Cartilage}

Regarding grades of cartilage damage, sensitivity increases the more severe the cartilage damage becomes (Table VI). Specificity was found to

Table V. Statistical data for lesions of the anterior cruciate ligament related to pathological changes (in \%)

\begin{tabular}{|lcc|}
\hline Parameter & $\begin{array}{c}\text { Grade } \\
\mathbf{0}+\mathbf{I}\end{array}$ & $\begin{array}{c}\text { Grade } \\
\text { II + III }\end{array}$ \\
\hline Specificity & 91.0 & 95.8 \\
\hline Sensitivity & 82.3 & 71.8 \\
\hline Negative predictive value & 95.7 & 96.2 \\
\hline Positive predictive value & 68.0 & 70.0 \\
\hline Accuracy & 89.4 & 93.0 \\
\hline
\end{tabular}

be high in all grades. Therefore minor damage of hyaline cartilage may be overlooked in MRI detection and often leads to false-negative results, whereas more severe damage will more likely be revealed.

For further differentiation, data were calculated for every single joint surface (Table VII). In assessment of cartilage lesions, specificity of $75-78 \%$ in the medial compartment was lower than in the lateral compartment, with about $90 \%$. In contrast, sensitivity was higher for the medial than for the lateral joint area. Specificity and sensitivity for the retropatellar surface were similar to data for the medial compartment.

\section{Discussion}

In the past, several authors proclaimed that $M R I$ is overused in evaluation of knee complaints, as an equally good or even better accuracy of diagnosis by careful clinical examination compared with MRI would be possible [24]. The question arises in which cases MRI is able to provide additional information to a thorough clinical exam-

Table VI. Results for cartilage lesions related to grading without differentiation of localization (in \%)

\begin{tabular}{|lcccc|}
\hline Parameter & Grade I & Grade II & Grade III & Grade IV \\
\hline Specificity & 98.3 & 88.5 & 96.1 & 96.0 \\
\hline Sensitivity & 6.5 & 28.9 & 37.6 & 68.8 \\
\hline Negative predictive value & 94.6 & 79.9 & 94.8 & 97.4 \\
\hline Positive predictive value & 18.8 & 44.0 & 44.6 & 58.5 \\
\hline Accuracy & 93.2 & 74.2 & 95.0 & 93.9 \\
\hline
\end{tabular}

Table VII. Overall results for cartilage lesions related to localization without grading (in \%)

\begin{tabular}{|lccccc|}
\hline Parameter & Retropatellar surface & Medial tibia & Medial femur & Lateral tibia & Lateral femur \\
\hline Specificity & 76.7 & 75.4 & 78.0 & 90.3 & 90.3 \\
\hline Sensitivity & 61.4 & 56.0 & 61.8 & 35.0 & 47.2 \\
\hline Negative predictive value & 61.2 & 64.8 & 54.9 & 70.6 & 74.1 \\
\hline Positive predictive value & 76.9 & 67.9 & 82.6 & 71.2 & 87.6 \\
\hline Accuracy & 68.2 & 66.1 & 67.9 & 80.9 \\
\hline
\end{tabular}


ination and if standard MRI is necessary before arthroscopy.

Reports in the literature revealed data which were assessed in a single imaging centre with one standardized MR scanner and specialized radiologists. In comparison, the data of our study were obtained with the interpretation of MRI reports by 23 outpatient radiology units without specialization in musculoskeletal diagnostics. The results must be considered under these conditions.

Our data show that for the following pathologies MRI from a radiology unit without specialization in musculoskeletal diagnostics is unable to provide an additional information gain or to recognize damage highly correctly: the medial and lateral meniscus, and the anterior cruciate ligament. In the literature on damage of the medial meniscus by means of detection by standardized MRI, sensitivity of $73-100 \%$ and specificity of $52-$ $100 \%$ were reported $[4,6,9]$. We found sensitivity of $58.3 \%$ for the anterior horn and $94.4 \%$ for the posterior horn of the medial meniscus. Specificity for the anterior horn of the medial meniscus was 93.1\% and for the posterior horn 46.2\%. However, with clinical examination, sensitivity of $50-92 \%$ and specificity of $55.6-97 \%$ for medial meniscal pathologies have previously been reported $[4,5,8$, 25]. For determination of lateral meniscal lesions by standardized MRI, sensitivity of $35-100 \%$ and specificity of $89-100 \%$ were reported $[4,6,9]$. In our study we found low sensitivity of $70.5 \%$ for the anterior and $62.2 \%$ for the posterior horn of the lateral meniscus, which is in accordance with data from other studies [16]. Specificity for the anterior horn of the lateral meniscus was $80.8 \%$ and for the posterior horn $82.3 \%$. For clinical evidence of lateral meniscus damage, similar high sensitivity of $54-92 \%$ and specificity of $90-96 \%$ were seen $[4,5,8]$. That shows that there is no information gain for meniscal tears with a preoperative MRI from a non-specialized radiology unit in addition to a thorough clinical examination. Even the question of whether meniscal repair is possible cannot be answered by preoperative MRI. Therefore, we see an indication for MRI with suspected meniscal lesions only in older patients to exclude Ahlbaeck's disease, but not in the young.

For presence of anterior cruciate ligament ruptures with standardized MRI, sensitivity of 44$100 \%$ and specificity of $89-99 \%$ were found in the literature $[4,7,9]$. We found sensitivity of $82.3 \%$ for grade $0+I$ changes and $71.8 \%$ for grade $I I+I I I$ ruptures of the anterior cruciate ligament. Specificity was $91 \%$ for grade $0+\mathrm{I}$ and $95.8 \%$ for grade II + III. Clinical examination also revealed good sensitivity of $77-97 \%$ and specificity of $88-100 \%[4,5]$. Therefore, in our opinion there is again no information gain for the arthroscopic procedure, and MRI from a non-specialized radiology unit can be waived. In relation to anterior cruciate ligament ruptures, we think that there is no need for MRI when surgery will take place immediately, because additional injuries will be assessed intraoperatively. But if anterior cruciate ligament reconstruction is planned to take place after an interval of several weeks, in our opinion MRI, which could be performed in non-specialized imaging centres, is necessary to rule out acute meniscal lesions or cartilage damage, which require immediate surgical treatment.

Therefore, we consider that preoperative MRI is helpful for arthroscopic planning if additional intra-articular damage, such as combined ruptures of the anterior and posterior cruciate ligament, is suspected. With planned delayed surgery of the anterior cruciate ligament, MRI, including that from a non-specialized radiology unit, can be very valuable because of poor accuracy of examination of a swollen knee for the diagnosis of associated injuries. However, in our view early surgical treatment within an inflammatory knee should not be considered unless there is meniscal injury or cartilage flake, which must be repaired immediately.

Another very important question is related to the diagnosis and therapy of cartilage damage in terms of prevention of early osteoarthritis. Cartilage lesions, detected by standardized MRI, are found in the literature with sensitivity in a very large range of $0-94 \%$ with a very high specificity of $91.4-99 \%$ [7, 9, 10]. In our study, we found that sensitivity increased with higher grade of cartilage damage, while specificity was similarly high at all grades. The literature shows that even with a standardized MRI intact or low-grade degenerated cartilage often gets rated to poorly [26]. But $\mathrm{MRI}$ correlates better with arthroscopic findings in cases of deep cartilage lesions (grade III + IV).

In our opinion, the data for specificity and sensitivity are insufficient to tell the patient preoperatively whether methods for cartilage regeneration have to be used. If this question should be answered, we think that the $T 2$ relaxation time or the dGEMRIC (delayed Gadolinium-Enhanced Magnetic Resonance Imaging of Cartilage) process will be necessary. Recent studies showed that three-dimensional standardized MRI scans promise the capability to detect a higher number of meniscal tears and especially early stages of osteoarthritis [27, 28]. Experimental standardized MRI studies with better technique, for example using higher magnetic flux density of 3 or 7 Tesla, in future can potentially bring an improvement of diagnosis of articular cartilage damage. But in the literature it has also already been pointed out that in regard to a remarkable number of false-positive and false-negative findings, the diagnostic value of standardized 3-Tesla MRI investigation should 
not be overestimated [29]. Furthermore, it was observed that the radiologist's experience seems to be more important than field strength [30].

A limitation of this study was that all patients who clinically presented pathology but which was not verified in MRI were not included in the study. That is why perhaps the false-negative rate is even higher, because patients with a false-negative MRI diagnosis were left out of the calculation.

In conclusion, in chronic knee pain, in our opinion, MRI, including that performed in non-specialized radiology units, should be performed before surgery in the elderly, in order not to miss pathologies that cannot be addressed by the operation or which need another surgical procedure (e.g. Ahlbaeck's disease). For acute injuries, from our point of view, MRI imaging, even when performed in outside imaging centres without musculoskeletal specialization, should be carried out in order not to overlook injuries which make surgery generally necessary or which require an operation immediately and not after a delay (e.g. cartilage flakes within primary traumatic patella dislocation, meniscal lesions with anterior cruciate ligament injuries).

\section{References}

1. Availabe at: http://ae.gelenk-klinik.com/de.

2. Crawford R, Walley G, Bridgman S, et al. Magnetic resonance imaging versus arthroscopy in the diagnosis of knee pathology, concentrating on meniscal lesions and ACL tears: a systematic review. Br Med Bull 2007; 84: 5-23.

3. Figueroa D, Calvo R, Vaisman A, et al. Knee chondral lesions: incidence and correlation between arthroscopic and magnetic resonance findings. Arthroscopy 2007; 23: 312-5.

4. Rayan F, Bhonsle S, Shukla DD. Clinical, MRI, and arthroscopic correlation in meniscal and anterior cruciate ligament injuries. Int Orthop 2009; 33: 129-32.

5. Nickinson R, Darrah C, Donell S. Accuracy of clinical diagnosis in patients undergoing knee arthroscopy. Int Orthop 2010; 34: 39-44.

6. Elvenes J, Jerome CP, Reikeras $\mathrm{O}$, et al. Magnetic resonance imaging as a screening procedure to avoid arthroscopy for meniscal tears. Arch Orthop Trauma Surg 2000; 120: 14-6.

7. Munk B, Madsen F, Lundorf E, et al. Clinical magnetic resonance imaging and arthroscopic findings in knees: a comparative prospective study of meniscus anterior cruciate ligament and cartilage lesions. Arthroscopy 1998; 14: 171-5.

8. Ryzewicz M, Peterson B, Siparsky PN, et al. The diagnosis of meniscus tears: the role of MRI and clinical examination. Clin Orthop Relat Res 2007; 455: 123-33.

9. Spiers AS, Meagher T, Ostlere SJ, et al. Can MRI of the knee affect arthroscopic practice? A prospective study of 58 patients. J Bone Joint Surg Br 1993; 75: 49-52.

10. Vallotton JA, Meuli RA, Leyvraz PF, et al. Comparison between magnetic resonance imaging and arthroscopy in the diagnosis of patellar cartilage lesions: a prospective study. Knee Surg Sports Traumatol Arthrosc 1995; 3: 157-62.
11. Biedert RM. Intrasubstance meniscal tears. Clinical aspects and the role of MRI. Arch Orthop Trauma Surg 1993; 112: 142-7.

12. Hughes RJ, Houlihan-Burne DG. Clinical and MRI considerations in sports-related knee joint cartilage injury and cartilage repair. Semin Musculoskelet Radiol 2011; 15: 69-88.

13. Helmark IC, Neergaard K, Krogsgaard MR. Traumatic knee extension deficit (the locked knee): can MRI reduce the need for arthroscopy? Knee Surg Sports Traumatol Arthrosc 2007; 15: 863-8.

14. Munshi M, Davidson M, MacDonald PB, et al. The efficacy of magnetic resonance imaging in acute knee injuries. Clin J Sport Med 2000; 10: 34-9.

15. Liodakis E, Hankemeier S, Jagodzinski M, et al. The role of preoperative MRI in knee arthroscopy: a retrospective analysis of 2,000 patients. Knee Surg Sports Traumatol Arthrosc 2009; 17: 1102-6.

16. Van Dyck P, Gielen J, D'Anvers J, et al. MR diagnosis of meniscal tears of the knee: analysis of error patterns. Arch Orthop Trauma Surg 2007; 127: 849-54.

17. Herman LJ, Beltran J. Pitfalls in MR imaging of the knee. Radiology 1988; 167: 775-81.

18. Fox MG. MR imaging of the meniscus: review, current trends, and clinical implications. Magn Reson Imaging Clin N Am 2007; 15: 103-23.

19. Sproule JA, Khan F, Rice JJ, et al. Altered signal intensity in the posterior horn of the medial meniscus: an MR finding of questionable significance. Arch Orthop Trauma Surg 2005; 125: 267-71.

20. Thornton DD, Rubin DA. Magnetic resonance imaging of the knee menisci. Semin Roentgenol 2000; 35: 217-30.

21. Mäurer J. Efficient Knee imaging. Georg-Thieme-Verlag 2004.

22. Outerbridge RE. The etiology of chondromalacia patellae. J Bone Joint Surg Br 1961; 43-B: 752-7.

23. Uhl M, Herget G, Altehoefer C. MRI of the hyaline joint cartilage. Arthroskopie 2001; 14: 109-13.

24. Yelland $M$. Review: clinical examination is often as accurate as magnetic resonance imaging for diagnosing meniscus tears. Evid Based Med 2007; 12: 151.

25. Jaddue DA, Tawfiq FH, Sayed-Noor AS. The utility of clinical examination in the diagnosis of medial meniscus injury in comparison with arthroscopic findings. Eur J Orthop Surg Traumatol 2010; 20: 389-92.

26. Spahn G, Wittig R, Kahl E, et al. Evaluation of cartilage defects in the knee: validity of clinical, magnetic-resonance-imaging and radiological findings compared with arthroscopy [German]. Unfallchirurg 2007; 110: 414-24.

27. Gluckert K, Kladny B, Blank-Schal A, et al. MRI of the knee joint with a 3-D gradient echo sequence. Equivalent to diagnostic arthroscopy? Arch Orthop Trauma Surg 1992; 112: 5-14.

28. Ohishi T, Takahashi M, Abe $M$, et al. The use of axial reconstructed images from three-dimensional MRI datasets for morphological diagnosis of meniscal tears of the knee. Arch Orthop Trauma Surg 2005; 125: 622-7.

29. von Engelhardt LV, Schmitz A, Pennekamp PH, et al. Diagnostics of degenerative meniscal tears at 3-Tesla MRI compared to arthroscopy as reference standard. Arch Orthop Trauma Surg 2008; 128: 451-6.

30. Krampla W, Roesel M, Svoboda K, et al. MRI of the knee: how do field strength and radiologist's experience influence diagnostic accuracy and interobserver correlation in assessing chondral and meniscal lesions and the integrity of the anterior cruciate ligament? Eur Radiol 2009; 19: 1519-28. 